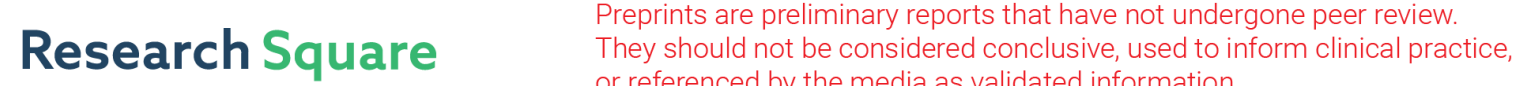 or referenced by the media as validated information. \\ Unified Phase Field Theory Based on the Interaction of Positive and Negative Magnetic poles and Analysis of ATLAS, CMS Experiment at the LHC
}

xianjin wu ( $\square$ physicswuxianjin@126.com )

School of Physics and Optoelectronic Engineering, Yangtze University

Research Article

Keywords:

Posted Date: December 28th, 2021

DOI: https://doi.org/10.21203/rs.3.rs-1190643/v1

License: (c) (1) This work is licensed under a Creative Commons Attribution 4.0 International License.

Read Full License 


\title{
Unified Phase Field Theory Based on the Interaction of Positive and Negative Magnetic poles and Analysis of ATLAS, CMS Experiment
}

\author{
at the $\mathrm{LHC}$ \\ Xianjin $\mathrm{Wu}$ \\ School of Physics and Optoelectronic Engineering, Yangtze University, Jingzhou, Hubei, China \\ Email:physicswuxianjin@126.com
}

\begin{abstract}
This article assumes that the elementary particle is a magnetic poles field formed by the interaction of positive and negative magnetic pole, believes that the gravity, the electromagnetic force, the strong force and the weak force are all produced by the interaction of positive and negative magnetic pole. The collision of the high-energy elementary particles appears as a strong force, and the decay of the high-energy elementary particles appears as a weak force, the cohesive force of the high-energy elementary particle magnetic pole field (the gravitational field) to its magnetic pole is the gravity, and the spin force of the high-energy elementary particle magnetic pole field in the external field (the gravitational field) is the electromagnetic force. This article discuss the high-energy proton-antiproton collision experiment based on the interaction of positive and negative magnetic pole, reveals the production mechanism of the protonium, tauium, muonium, positronium, three generation of leptons and neutrinos, and final state. This article explains unify of the strong force, weak force, electromagnetic force and gravity with unified phase field theory, and tested with the data of ATLAS and CMS experiment at the LHC. The data of ATLAS and CMS experiment at the LHC is completely consistent with the calculated data of the phase field curvature tensor equation; Differential geometric variables are covariant with physical variables; The Lagrangian function of Einstein's mass-energy equation, the Lagrangian function of Schrodinger particle differential motion wave function based on the theory of relativity, the Lagrangian density of Young-Mills gauge field equation, and the planets phase difference momentum-energy tensor of the curvature tensor equation is completely consistent in the high-energy proton-antiproton collision experiment. These fully prove that the unified phase field theory is more in line with the physical reality of the high-energy proton-antiproton collision experiment.
\end{abstract}

\section{Introduction}

In 1963, Gellman and Zweig 1,2,3,4 independently proposed that hadrons are composed of quarks. They believed that these hadrons are composed of different combinations of u quarks, $d$ quarks, and s quarks, the "The Eightfold Way" according to the theory of the SU(3) group symmetry. In 1957, Schwinger ${ }^{5}$ predicted that the intermediate boson $W_{+} 、 W_{-}$would act as a mediator like photons, and directly unify the weak interaction and electromagnetic interaction, based on the Yang-Mills gauge field theory ${ }^{6}$ and the Higgs mechanism of spontaneous symmetry breaking ${ }^{7,8,9}$. In 1967 , Weinberg ${ }^{10}$ described the unity of weak interaction and electromagnetic interaction, based on the idea of strict gauge symmetry but 
spontaneously broken, after further research by Glashaw, Weinberg, and Salam. In 1973, Gross et al. ${ }^{11}$ discussed non-Abelian gauge field theory of the strong interaction under SU(3) the chromatic gauge group. They believed that the interacting mediators were massless gluons, and found that the value of the function was negative. They found that Hadron in this gauge field have the properties of asymptotically free, thus the quantum chromodynamics (QCD) based on SU(3) the chromatic gauge symmetry group is established. Standard Model theory for explaining the unity of electromagnetic interaction, strong interaction and weak interaction was established through the efforts of Glasow and others.

In 1973, Li Zhengdao ${ }^{12}$ proposed that it is possible to realize the transformation from the hadron phase to the quark matter phase through the relativistic heavy ion collision experiment. Thus, people began to produce quark gluon plasma (QGP) through heavy ion collision experiments, and to search various particles predicted in the Standard Model ${ }^{13,14,15,16}$. In 1973, the CERN ${ }^{17,18}$ proton-antiproton collider found the tracks of neutral flow through heavy liquid bubble chambers. It is believed that the neutral intermediate boson $\mathrm{Z}$, which propagates weak force in the scattering process of neutrinos and electrons and nucleons. In 1974, Ding Zhaozhong and Richter ${ }^{19,20}$ discovered charm quark C. In 1977 , Lederman and his colleagues at Fermi National Laboratory ${ }^{21}$ discovered $\mathrm{T}$ particles (similar to $\mathrm{J} / \Psi$ particles, particles composed of bottom quarks). In 1979, the high-energy electron-positron collisions at Betra Hamburg, Germany discovered the phenomenon of three jets ${ }^{22}$, showing the existence of gluons. In 1983, CERN ${ }^{23}$ found the intermediate boson W and Z. In 1995, the CDF and Dø Collaboration of Fermi National Laboratory ${ }^{24,25}$ detected top quarks in high-energy proton-antiproton collisions. In 2000, Fermi National Laboratory discovered T neutrinos ${ }^{26}$. On July 4, 2012, ATLAS and CMS collaboration on the LHC jointly announced the discovery of a new boson ${ }^{27,28}$, and the results showed that it conformed to the characteristics of the Higgs boson. So far, people have found all the particles predicted by the Standard Model.

Searching for quarks, heavy bosons, gluons, and Higgs particles through high-energy particle collision experiments is actually looking for hadrons for quantum chromodynamics calculations. QDP (quark and gluon plasma) was produced through high-energy particle collision experiment, and the corresponding signals with "jet quenching", "J/ $\psi$ particle depression", and "strange particles relative yield increase" were found Hadrons, and calculate the corresponding particles through the quantum chromodynamics.

Various high-energy particle collision experiments are scientific, and the experimental results and descriptions are credible, but it is very difficult to explain with the relevant theories of the Standard Model. This article assumes that the elementary particle is a magnetic poles field formed by the interaction of positive and negative magnetic pole, believes that gravity, electromagnetic force, strong force and weak force are all produced by the interaction of positive and negative magnetic pole. The collision of the high-energy elementary particles appears as a strong force, and the decay of the high-energy elementary particles appears as a weak force, the cohesive force of the high-energy elementary particle magnetic pole field (the gravitational field) to its magnetic pole is the gravity, and the spin force of the high-energy elementary particle magnetic pole field in the external field (the gravitational field) is the electromagnetic force. This article discuss the high-energy proton-antiproton collision experiment based on the interaction of positive and negative magnetic pole, reveals the production mechanism of the protonium, tauium, muonium, positronium, three generation of leptons and neutrinos, and final state. This article explains unify of the strong force, weak force, electromagnetic force and gravity with unified phase field theory, and tested with the data of ATLAS 
and CMS experiment at the LHC. The data of ATLAS and CMS experiment at the LHC is completely consistent with the calculated data of the phase field curvature tensor equation; Differential geometric variables are covariant with physical variables; The Lagrangian function of Einstein's mass-energy equation, the Lagrangian function of Schrodinger particle differential motion wave function based on the theory of relativity, the Lagrangian density of Young-Mills gauge field equation, and the planets phase difference momentum-energy tensor of the curvature tensor equation is completely consistent in the high-energy proton-antiproton collision experiment. These fully prove that the unified phase field theory is more in line with the physical reality of the high-energy proton-antiproton collision experiment.

\section{Main}

\section{Data of ATLAS and CMS experiment}

Data of ATLAS experiment ${ }^{27}$. The datasets used correspond to integrated luminosities of approximately $4.8 \mathrm{fb}^{-1}$ collected at $\sqrt{s}=7 \mathrm{TeV}$ in 2011 and $5.8 \mathrm{fb}^{-1}$ at $\sqrt{s}=8 \mathrm{TeV}$ in 2012 . Individual searches in the channels $H \rightarrow Z Z^{(*)} \rightarrow 4 \ell, H \rightarrow \gamma \gamma$ and $H \rightarrow W W^{(*)} \rightarrow e v \mu v$ in the $8 \mathrm{TeV}$ data are combined with previously published results of searches for $H \rightarrow Z Z^{(*)}, W W^{(*)}, b . b$ and $\tau^{+} \tau^{-}$in the $7 \mathrm{TeV}$ data and results from improved analyses of the $H \rightarrow Z Z^{(*)} \rightarrow 4 \ell$ and $H \rightarrow \gamma \gamma$ channels in the $7 \mathrm{TeV}$ data. Clear evidence for the production of a neutral boson with a measured mass of $126.0 \pm 0.4$ (stat) \pm 0.4 (sys) $\mathrm{GeV}$ is presented. This observation, which has a significance of 5.9 standard deviations, corresponding to a background fluctuation probability of $1.7 \times 10^{-9}$, is compatible with the production and decay of the Standard Model Higgs boson.

Table 1 | Summary of the individual channels entering the combination. The transition points between separately optimised $m_{H}$ regions are indicated where applicable. In channels sensitive to associated production of the Higgs boson, $\mathrm{V}$ indicates a $W$ or $Z$ boson. The symbols $\otimes$ and $\oplus$ represent direct products and sums over sets of selection requirements, respectively.

\begin{tabular}{|c|c|c|c|c|}
\hline $\begin{array}{l}\text { Higgs boson } \\
\text { decay }\end{array}$ & $\begin{array}{c}\text { Subsequet } \\
\text { decay }\end{array}$ & Sub-channels & $\mathrm{m}_{H}$ range $[\mathrm{GeV}]$ & $\int L d t\left[f b^{-1}\right]$ \\
\hline \multicolumn{5}{|l|}{$2011 \sqrt{\mathrm{s}}=7 \mathrm{TeV}$} \\
\hline \multirow[t]{3}{*}{$\mathrm{H} \rightarrow \mathrm{ZZ}^{(*)}$} & $4 \ell$ & $\{4 \mathrm{e}, 2 \mathrm{e} 2 \mu, 2 \mu 2 \mathrm{e}, 4 \mu\}$ & $110-600$ & 4.8 \\
\hline & $\ell \ell v \bar{v}$ & $\{$ ee, $\mu \mu\} \otimes\{$ low,high pile-up $\}$ & $200-280-600$ & 4.7 \\
\hline & $\ell \ell q \bar{q}$ & $\{b$-tagged, untagged $\}$ & $200-300-600$ & 4.7 \\
\hline$H \rightarrow \gamma \gamma$ & - & 10 categories $\left\{p_{T_{t}} \otimes \eta_{\gamma} \otimes\right.$ conversion $\} \oplus\{2$-jet $\}$ & $110-150$ & 4.8 \\
\hline \multirow[t]{2}{*}{$H \rightarrow W W^{(*)}$} & $\ell v \ell v$ & $\{$ ee,e $\mu / \mu \mathrm{e}, \mu \mu\} \otimes\{0$-jet,2-jet $\} \otimes\{$ low, high pile-up $\}$ & $110-200-300-600$ & 4.7 \\
\hline & $\ell v q q^{\prime}$ & $\{\mathrm{e}, \mu\} \otimes\{0$-jet, 1 -jet, 2 -jet $\}$ & $300-600$ & 4.7 \\
\hline \multirow[t]{4}{*}{$\mathrm{H} \rightarrow \tau \tau$} & $\tau_{\text {lep }} \tau_{\text {lep }}$ & $\{\mathrm{e}, \mu\} \otimes\{0$-jet $\} \oplus\{\ell \ell\} \otimes\{1$-jet, 2-jet , V H $\}$ & $110-150$ & 4.7 \\
\hline & $\tau_{\text {lep }} \tau_{\text {had }}$ & $\{\mathrm{e}, \mu\} \otimes\{0$-jet $\} \otimes\left\{E_{T}^{m i s s}<20 \mathrm{GeV}, E_{T}^{m i s s}>20 \mathrm{GeV}\right\}$ & $110-150$ & 4.7 \\
\hline & & $\oplus\{\mathrm{e}, \mu\} \otimes\{1$-jet $\} \oplus\{\ell\} \otimes\{2$-jet $\}$ & & \\
\hline & $\tau_{\text {had }} \tau_{\text {had }}$ & $\{1$-jet $\}$ & $110-150$ & 4.7 \\
\hline $\mathrm{VH} \rightarrow \mathrm{Vbb}$ & $Z \rightarrow v v$ & $E_{T}^{m i s s} \in\{120-160,160-200, \geq 200 \mathrm{GeV}\}$ & $110-130$ & 4.6 \\
\hline
\end{tabular}




\begin{tabular}{c|c|l|l|c}
\hline & $W \rightarrow \ell v$ & $p_{T}^{W} \in\{<50,50-100,100-200, \geq 200 \mathrm{GeV}\}$ & $110-130$ & 4.7 \\
& $Z \rightarrow \ell \ell$ & $p_{T}^{Z} \in\{<50,50-100,100-200, \geq 200 \mathrm{GeV}\}$ & $110-130$ & 4.7 \\
$2012 \sqrt{\mathrm{s}}=8 \mathrm{TeV}$ & & & & \\
$\mathrm{H} \rightarrow \mathrm{ZZ}{ }^{(*)}$ & $4 \ell$ & $\{4 \mathrm{e}, 2 \mathrm{e} 2 \mu, 2 \mu 2 \mathrm{e}, 4 \mu\}$ & $110-600$ & 5.8 \\
$H \rightarrow \gamma \gamma$ & - & 10 categories $\left\{p_{T_{t}} \otimes \eta_{\gamma} \otimes\right.$ conversion $\} \oplus\{2$-jet $\}$ & $110-150$ & 5.9 \\
$H \rightarrow W W^{(*)}$ & $e v \mu \nu$ & $\{\mathrm{e} \mu, \mu \mathrm{e}\} \otimes\{0$-jet,1-jet,2-jet $\}$ & $110-200$ & 5.8 \\
\hline
\end{tabular}

Data of CMS experiment ${ }^{28}$. Results are presented from searches for the standard model Higgs boson in proton-proton collisions at $\sqrt{s}=7$ and $8 \mathrm{TeV}$ in the Compact Muon Solenoid experiment at the LHC, using data samples corresponding to integrated luminosities of up to $5.1 \mathrm{fb}^{-1}$ at $7 \mathrm{TeV}$ and $5.3 \mathrm{fb}^{-1}$ at 8 $\mathrm{TeV}$. The search is performed in five decay modes: $\gamma \gamma, \mathrm{ZZ}, \mathrm{W}^{+} \mathrm{W}^{-}, \tau^{+} \tau^{-}$, and $b \bar{b}$. An excess of events is observed above the expected background, with a local significance of 5.0 standard deviations, at a mass near $125 \mathrm{GeV}$, signalling the production of a new particle. The expected significance for a standard model Higgs boson of that mass is 5.8 standard deviations. The excess is most significant in the two decay modes with the best mass resolution, $\gamma \gamma$ and ZZ; a fit to these signals gives a mass of $125.3 \pm 0.4$ (stat.) \pm 0.5 (syst.) GeV. The decay to two photons indicates that the new particle is a boson with spin different from one.

Table 2 | The number of selected events, compared to the expected background yields and expected number of signal events $\left(m_{H}=125 \mathrm{GeV}\right)$ for each final state in the $\mathrm{H} \rightarrow \mathrm{ZZ}$ analysis. The estimates of the $\mathrm{Z}+\mathrm{X}$ background are based on data. These results are given for the mass range from 110 to $160 \mathrm{GeV}$.

\begin{tabular}{|c|c|c|c|c|}
\hline channel & $4 e$ & $4 \mu$ & $2 e 2 \mu$ & $4 \ell$ \\
\hline ZZ background & $2.7 \pm 0.3$ & $5.7 \pm 0.6$ & $7.2 \pm 0.8$ & $15.6 \pm 1.4$ \\
\hline $\mathrm{Z}+\mathrm{X}$ & $1.2_{-0.8}^{+1.1}$ & $0.9_{-0.6}^{+0.7}$ & $2.3_{-1.4}^{+1.8}$ & $4.4_{-1.7}^{+2.2}$ \\
\hline All background $\left(110<m_{4 \ell}<160 \mathrm{GeV}\right)$ & $4.0 \pm 1.0$ & $6.6 \pm 0.9$ & $9.7 \pm 1.8$ & $20 \pm 3$ \\
\hline Observed $\left(110<m_{4 \ell}<160 \mathrm{GeV}\right)$ & 6 & 6 & 9 & 21 \\
\hline $\operatorname{Signal}\left(m_{H}=125 \mathrm{GeV}\right)$ & $1.36 \pm 0.22$ & $2.74 \pm 0.32$ & $3.44 \pm 0.44$ & $7.54 \pm 0.78$ \\
\hline All background (signal region) & $0.7 \pm 0.2$ & $1.3 \pm 0.1$ & $1.9 \pm 0.3$ & $3.8 \pm 0.5$ \\
\hline Observed (signal region) & 1 & 3 & 5 & 9 \\
\hline
\end{tabular}

The data of ATLAS and CMS experiment show that $m_{H}$ equivalent to the sum of the relativistic masses of the corresponding scattered particle produced from the energy field of high-energy proton-antiproton collisions, such as determining the mass of the four leptons, $m_{H}=125 \mathrm{GeV}$. The participation of bosons such as $\mathrm{W}$ and $\mathrm{Z}$ is not required in the high-energy proton-antiproton collisions based on the interaction of positive and negative magnetic poles. The relativistic masses of the scattering particles is not given by the Higgs boson, but is inherited from the previous generation particles. The Higgs particle invariant mass spectrum used in the ATLAS and CMS experiment should be interpreted as the invariant mass spectrum of scattered particle produced from the High-energy particle collision point. This is more in line with physical reality.

The data of high-energy proton-antiproton collisions based on the interaction of positive and negative magnetic poles 
How the scattering particles are produced cannot be clearly explained by quantum chromodynamics. High-energy particles based on the interaction of positive and negative magnetic poles have multi-level collision and decay mechanisms. The high-energy proton-antiproton collisions at the collision point to produce secondary particles, and the secondary particles collide in the high-energy field of the collision to produce scattered particles (or jets). The scattered particles enter the detector and collide with the atomic nucleus or outer electrons to produce the final state. This is used to explain ATLAS and CMS experimental data, which is more in line with physical reality.

Table 3 | Correspondence between scattered particle in the energy field of $7 \mathrm{TeV}$ proton collisions and decay particles in the energy field of the detector

\begin{tabular}{|c|c|c|c|c|}
\hline \multicolumn{2}{|c|}{ In the collisions point } & \multirow{2}{*}{$\begin{array}{c}\text { The scattered particle } \\
\text { Energy range }\end{array}$} & \multicolumn{2}{|r|}{ In the detector } \\
\hline $\begin{array}{c}\text { Collision } \\
\text { mode }\end{array}$ & $\begin{array}{c}\text { Decay } \\
\text { particles }\end{array}$ & & $\begin{array}{c}\text { Collision } \\
\text { mode }\end{array}$ & decay particles \\
\hline$p^{+} \Leftrightarrow p^{-}$ & $p_{p p}^{+}, p_{p p}^{-}$ & $2228 \mathrm{GeV}>p_{p p}^{+,-}>354.6 \mathrm{GeV}$ & & \\
\hline$p^{+} \Leftrightarrow p^{-}$ & $\tau^{+} v_{\tau}^{-}, \tau^{-} v_{\tau}^{+}$ & $948.18 \mathrm{GeV}>\tau>150.91 \mathrm{GeV}$ & & \\
\hline $\begin{array}{l}p_{p p}^{+} \Leftrightarrow p_{p p}^{-} \\
p_{p p}^{+,-} \Leftrightarrow p^{-,+}\end{array}$ & $p^{+}, p^{-}$ & $177.312077 \mathrm{GeV}>p>\cdots$ & $p^{+}, p^{-} \Leftrightarrow N$ & $0-$ jet, $1-$ jet, Baryon \\
\hline$\tau^{+} \Leftrightarrow \tau^{-}$ & $\tau_{\tau \tau}^{+}, \tau_{\tau \tau}^{-}$ & $301.814326 \mathrm{GeV}>\tau_{\tau \tau}^{+,-}>\cdots$ & $\tau_{t \tau}^{+}, \tau_{t \tau}^{-} \Leftrightarrow N$ & Meson, $1-j e t, 2-j e t, \mu^{+} v_{\mu}^{-}, \mu^{-} v_{\mu}^{+}$ \\
\hline $\begin{array}{l}\tau^{+} \Leftrightarrow \tau^{-} \\
\tau^{+,-} \Leftrightarrow p^{-,+}\end{array}$ & $\mu^{+} v_{\mu}^{-}, \mu^{-} v_{\mu}^{+}$ & $141.041527 \mathrm{GeV}>\mu>\cdots$ & $\mu^{+}, \mu^{-} \Leftrightarrow e$ & $e^{+} v_{e}^{-}, e^{-} v_{e}^{+}, 0-j e t, 1-j e t$ \\
\hline$\mu^{+} \Leftrightarrow \mu^{-}$ & $e^{+} v_{e}^{-}, e^{-} v_{e}^{+}$ & $22.3997427 \mathrm{GeV}>e>\cdots$ & $e^{+}, e^{-} \Leftrightarrow e$ & $0-j e t, 1-j e t, e_{e e}^{+}, e_{e e}^{-}, e^{+}, e^{-}$ \\
\hline$\mu^{+} \Leftrightarrow \mu^{-}$ & $\mu_{\mu \mu}^{+}, \mu_{\mu \mu}^{-}$ & $44.894912 G e V>\mu_{\mu \mu}^{+,-}>\cdots$ & $\mu_{\mu \mu}^{+}, \mu_{\mu \mu}^{-} \Leftrightarrow e$ & $\mu^{+}, \mu^{-}, 1-j e t, 2-j e t, e^{+} v^{-}, e^{-} v^{+}$ \\
\hline
\end{tabular}

In the table, $" \Leftrightarrow "$ represents the inelastic collision of high-energy particle, $p_{p p}^{+}, p_{p p}^{-}$represents protonium, anti-protonium. For example, protonium is composed of proton and antiproton colliding and merging (Fig.b,c). $p_{p p}^{+,-}$represents protonium or anti-protonium; $\tau_{\tau t}^{+}, \tau_{\tau t}^{-}$represents tauium, anti-tauium; $\mu_{\mu \mu}^{+}, \mu_{\mu \mu}^{-}$represents muonium, anti-muonium; $e_{e c}^{+}, e_{c e}^{-}$represents positronium, antipositronium; $p^{-,+}$represents incident proton or antiproton; $\tau^{+,-}$represents tau or anti-tau; ${ }_{N}$ represents atomic nucleus. Relativistic energy (or mass) of decay particles

$$
E_{d}=E_{c} / 2 \pi,
$$

Where $E_{c}$ is the energy of the collide particle.

Table 4 | Data of energy and radius of particles scattered in the energy field of ${ }_{7 \mathrm{TeV}}$ and $8 \mathrm{TeV}$ proton collision

\begin{tabular}{|c|c|c|c|c|c|c|c|}
\hline \multirow{2}{*}{$\begin{array}{l}\text { Collision } \\
\text { mode }\end{array}$} & \multirow{2}{*}{$\begin{array}{c}\text { Decay } \\
\text { particles }\end{array}$} & \multirow{2}{*}{$\begin{array}{c}7 \mathrm{TeV} \\
\text { Collision } \\
\text { point }\end{array}$} & \multirow{2}{*}{$\begin{array}{c}8 \mathrm{TeV} \\
\text { Collision } \\
\text { point }\end{array}$} & \multicolumn{2}{|c|}{ The detector $(7 \mathrm{TeV})$} & \multicolumn{2}{|c|}{ The detector $(8 \mathrm{TeV})$} \\
\hline & & & & $\begin{array}{r}\text { Energy } \\
(\mathrm{GeV})\end{array}$ & $\begin{array}{c}\text { Radius } \\
(\mathrm{m}) \\
\end{array}$ & $\begin{array}{r}\text { Energy } \\
(\mathrm{GeV}) \\
\end{array}$ & $\begin{array}{c}\text { Radius } \\
(\mathrm{m})\end{array}$ \\
\hline$p^{+} \Leftrightarrow$ & $p_{p p}^{-}$ & $2228.17 \mathrm{GeV}$ & $2546.479 \mathrm{GeV}$ & --- & & --- & \\
\hline $\begin{array}{l}p_{p p}^{+} \Leftrightarrow p_{p p}^{-} \\
p_{p p}^{+,-} \Leftrightarrow p^{-,+}\end{array}$ & $p^{+}, p^{-}$ & $177.312 \mathrm{GeV}$ & $202.6424 \mathrm{GeV}$ & 175.548 & $5.51367 \times 10^{-15} \mathrm{~m}$ & 200.878 & $5.27143 \times 10^{-15} \mathrm{~m}$ \\
\hline$p^{+} \Leftrightarrow p^{-}$ & $\begin{array}{l}\tau^{+}, \tau^{-} \\
v_{-}^{+}, v_{-}^{-}\end{array}$ & $\begin{array}{l}948.1777 \mathrm{GeV} \\
165.907 \mathrm{GeV}\end{array}$ & $\begin{array}{l}1107.333 \mathrm{GeV} \\
165.907 \mathrm{GeV}\end{array}$ & $\begin{array}{l}--- \\
165.907\end{array}$ & $1.51407 \times 10^{-16} \mathrm{~m}$ & $\begin{array}{l}--- \\
165.907\end{array}$ & $1.51407 \times 10^{-16} \mathrm{~m}$ \\
\hline$\tau^{+} \Leftrightarrow \tau^{-}$ & $\tau_{t r}^{+}, \tau_{\tau t}^{-}$ & $301.814 \mathrm{GeV}$ & $352.4749 \mathrm{GeV}$ & 295.24 & $4.66364 \times 10^{-15} \mathrm{~m}$ & 345,9 & $4.39801 \times 10^{-15} \mathrm{~m}$ \\
\hline
\end{tabular}




\begin{tabular}{l|l|l|l|l|l|l|l}
\hline$\tau^{+} \Leftrightarrow \tau^{-}$ & $\mu^{+}, \mu^{-}$ & $141.0415 \mathrm{GeV}$ & $166.3718 \mathrm{GeV}$ & 140.619 & $5.93688 \times 10^{-15} \mathrm{~m}$ & 165.949 & $5.618 \times 10^{-15} \mathrm{~m}$ \\
$\tau^{+,-} \Leftrightarrow p^{-,+}$ & $v_{\mu}^{+}, v_{\mu}^{-}$ & $9.86564 \mathrm{GeV}$ & $9.86564 \mathrm{GeV}$ & 9.86564 & $3.87905 \times 10^{-16} \mathrm{~m}$ & 9.86564 & $3.87905 \times 10^{-16} \mathrm{~m}$ \\
$\mu^{+} \Leftrightarrow \mu^{-}$ & $\mu_{\mu \mu}^{+}, \mu_{\mu \mu}^{-}$ & $44.89491 \mathrm{GeV}$ & $52.9578 \mathrm{GeV}$ & 44.6095 & $8.70491 \times 10^{-15} \mathrm{~m}$ & 52.6728 & $8.2359 \times 10^{-15} \mathrm{~m}$ \\
& $e^{+}, e^{-}$ & $22.39974 \mathrm{GeV}$ & $26.431186 \mathrm{GeV}$ & 22.399 & $1.09521 \times 10^{-14} \mathrm{~m}$ & 26.4305 & $1.03643 \times 10^{-14} \mathrm{~m}$ \\
$\mu^{+} \Leftrightarrow \mu^{-}$ & $v_{e}^{+}, v_{e}^{-}$ & $47.7135 \mathrm{MeV}$ & $47.7135 \mathrm{MeV}$ & 0.04771 & $2.29379 \times 10^{-15} \mathrm{~m}$ & 0.04771 & $2.29379 \times 10^{-15} \mathrm{~m}$ \\
\hline
\end{tabular}

The scattered particle are incident in the detector and collide with the nucleus or outer electrons, take different paths in the 3.8T magnetic field, and release the corresponding energy (magnetic poles). The scattering angle of the scattering particles is different, and the path length is different. Take the CMS detector as an example. Set up that the path length of the electrons and the muonium incident in the electromagnetic calorimeter to collide with the outer electrons is $1.5 \mathrm{~m}$ and $1.35 \mathrm{~m}$, the path length of the proton and the tauium incident in the hadron calorimeter to collide with the nucleus is $1.88 \mathrm{~m}$ and $1.85 \mathrm{~m}$, and the path length of the muon incident in the muon detector to collide with argon gas is $4 \mathrm{~m}$. The interaction between the scattered particle and the 3.8T magnetic field releases the corresponding energy and reaches the collision point of corresponding detector. The energy released by the elastic collision of scattered particle at the trigger point of the detector is ignored in the table. The radius of positive magnetic pole ring of the elementary particle

$$
r_{\text {ring }}=\alpha r \text {, }
$$

Where $\alpha$ is the fine-structure constant, $r$ is the radius of the elementary particle. For example, the radius of positive magnetic pole ring of $7 \mathrm{TeV}$ high-energy proton is $1.17771171785662 \times 10^{-17}$.

The particle the relativistic masses (the energy $E$ ) is calculated by the equation (4) and the equation (6). The particle radius $(r)$ is calculated by the equation (9). The data of the table 4 show that the sum of the relativistic masses (the energy) of the corresponding scattering particles equivalent to the masses of corresponding Higgs boson in the table 1,2.

\section{Data of High-energy particle collisions to verify the unified phase field theory}

The high-energy particles collide in the collision energy field, and scattered particle collide in the detector energy field, all moving on a certain space-time curve. Every point on the space-time curve can be described by the unified phase field theory. High-energy proton, high-energy protonium, high-energy tau, from collision to decay, or from strong interaction to weak interaction, can be described by the unified phase field curvature tensor equation (equation (11)). Take data of $7 \mathrm{TeV}$ proton collision as an example (Table.5, 6).

Table 5.1 | Strong interaction data of high-energy particle collision at the collision point

\begin{tabular}{l|c|c|l|l|l|l}
\hline \multirow{2}{*}{ particles } & $\lambda_{m}$ & $m$ & \multicolumn{1}{c|}{$v$} & $E, E_{c}$ & $F$ & $\phi_{\mu \nu}$ \\
\cline { 2 - 7 } & $10^{-12} m$ & $M e V$ & $m / s$ & $M e V$ & $N$ & $\mathrm{~m}^{-1}$ \\
\hline$p^{+}, p^{-}$ & 4.852621155 & 938.272046 & 299792455.306902 & 6999061.959 & $1.9603782 \times 10^{-35}$ & $2.623138 \times 10^{5}$ \\
$p_{p p}^{+}, p_{p p}^{-}$ & 4.8526245097 & 1876.54409 & 299792351.68073 & 2226292.685 & $6.235658 \times 10^{-36}$ & $1.206091 \times 10^{6}$ \\
$\tau^{+}, \tau^{-}$ & 4.8526381084 & 1776.82 & 299791931.6211 & 946400.891 & $2.6507918 \times 10^{-36}$ & $3.76423 \times 10^{6}$ \\
\hline
\end{tabular}

In the table, $\lambda_{m}, m, v, E, E_{c}, F$ and respectively represent the motion wavelength, mass, velocity, energy and gravity of high-energy particle. $\phi_{u v}$ represent the curvature tensor when particles interact strongly. The masses of various elementary particles in the article use the recommended values of the 2012 International Particle Data Group ${ }^{29}$.

Table 5.2 | Strong interaction data of high-energy particle collision at the collision point

\begin{tabular}{c|c|c|c|c|c|c}
\hline$\varphi_{\mu \nu}$ & $R_{\mu \nu}$ & $r$ & $R$ & $r_{F}$ & $M_{\mu \nu}$ & $T_{\mu \nu}$ \\
\hline $\mathrm{m}$ & $\mathrm{m}^{-1}$ & $\mathrm{~m}$ & $\mathrm{~m}^{-2}$ & $\mathrm{~m}$ & $\mathrm{MeV}, \rho_{p} / \mathrm{m}^{3}$ & $\mathrm{MeV}, \rho_{p} / \mathrm{m}^{3}$ \\
\hline $1.36417 \times 10^{-15}$ & $5.246276 \times 10^{5}$ & $1.613889 \times 10^{-15}$ & $3.84576 \times 10^{20}$ & $7.21147 \times 10^{-11}$ & 5916096.113 & $1.05464 \times 10^{-23}$ \\
\hline
\end{tabular}




\begin{tabular}{l|l|l|l|l|l|l}
\hline $1.99796 \times 10^{-15}$ & $2.412181 \times 10^{6}$ & $2.363689 \times 10^{-15}$ & $1.20733 \times 10^{21}$ & $4.07008 \times 10^{-11}$ & 1881818.059 & $3.35465 \times 10^{-24}$ \\
$2.65627 \times 10^{-15}$ & $7.528459 \times 10^{6}$ & $3.142509 \times 10^{-15}$ & $2.83422 \times 10^{21}$ & $2.65643 \times 10^{-11}$ & 799964.7678 & $1.42607 \times 10^{-24}$ \\
\hline
\end{tabular}

In the table, $R_{u v} 、 r 、 R 、 r_{F} 、 \varphi_{u v} 、 M_{u v} 、 T_{u v}$ are the relevant variables of the curvature tensor equation. $\varphi_{u v}$ is the phase difference between the collided particle and the scattered particle. $M_{\mu v}$ is the energy $\left(E_{f}\right)$ released when the high-energy particles collide.

The wavelength $\left(\lambda_{m}\right)$ of the particle's motion is calculated by equation (5). The Ricci curvature scalar $R=2 K=2 / r_{F}^{2}$ of the particle, the Ricci curvature tensor $R_{\mu v}=R \varphi_{\mu v}$ of the particle. The particle energy ( $E$ or $E_{c}$ ) is calculated by the equation (4) and the equation (6). The particle gravitational energy ( $F$ or $f$ ) is calculated by the equation (7). The particle radius $(r)$ is the phase $\left(r_{\varphi}\right)$ of the particle on the space-time curve and is calculated by the equation (9). The radius of the gravitational field, that is, the maximum radius when high-energy particles collide

$$
r_{F}=\sqrt{G\left(m+m_{m}\right)^{2} / F},
$$

Let $M_{\mu \nu}$ be the Lagrangian density, $M_{\mu v}$ is the energy difference between the collided particle and the scattered particle, which can be calculated respectively by equation (12), equation (13), and equation (14). $T_{\mu v}$ is the gravitational force difference between the collided particle and the scattered particle, which can be calculated by equation (15).

Table 6.1 Weak interaction data of the decay of high-energy particle at the collision point

\begin{tabular}{l|c|c|c|c|c|c}
\hline \multirow{2}{*}{ particles } & $\lambda_{m}$ & $m$ & $v$ & $E, E_{c}$ & $F$ & $\phi_{\mu \nu}$ \\
\cline { 2 - 7 } & $10^{-12} m$ & $M e V$ & $m / s$ & $M e V$ & $N$ & $\mathrm{~m}^{-1}$ \\
\hline$p^{+}, p^{-}$ & 4.852633411 & 938.272046 & 299792076.7222 & 1112307.768 & $3.1154834 \times 10^{-36}$ & $3.592779 \times 10^{6}$ \\
$p_{p p}^{+}, p_{p p}^{-}$ & 4.8526227909 & 1876.54409 & 299792404.77431 & 354412.8256 & $9.9268145 \times 10^{-37}$ & $1.654746 \times 10^{7}$ \\
$\tau^{+}, \tau^{-}$ & 4.8526234466 & 1776.82 & 299792384.51835 & 150801.5006 & $4.2238227 \times 10^{-37}$ & $5.169288 \times 10^{7}$ \\
\hline
\end{tabular}

Table 6.2 | Weak interaction data of the decay of high-energy particle at the collision point

\begin{tabular}{c|c|c|c|c|c|c}
\hline$g_{\mu v}$ & $R_{\mu v}$ & $r$ & $R$ & $r_{F}$ & $M_{\mu v}$ & $T_{\mu v}$ \\
\hline $\mathrm{m}$ & $\mathrm{m}^{-1}$ & $\mathrm{~m}$ & $\mathrm{~m}^{-2}$ & $\mathrm{~m}$ & $M e V, \rho_{p} / \mathrm{m}^{3}$ & $\mathrm{MeV}, \rho_{p} / \mathrm{m}^{3}$ \\
\hline $2.97806 \times 10^{-15}$ & $7.185559 \times 10^{6}$ & $2.978062 \times 10^{-15}$ & $2.41283 \times 10^{21}$ & $2.87907 \times 10^{-11}$ & 1112307.768 & $1.98287 \times 10^{-24}$ \\
$4.36164 \times 10^{-15}$ & $3.309492 \times 10^{7}$ & $4.361645 \times 10^{-15}$ & $7.58772 \times 10^{21}$ & $1.62353 \times 10^{-11}$ & 354412.8256 & $6.31799 \times 10^{-25}$ \\
$5.79878 \times 10^{-15}$ & $1.033858 \times 10^{8}$ & $5.798778 \times 10^{-15}$ & $1.78289 \times 10^{22}$ & $1.05914 \times 10^{-11}$ & 150801.5006 & $2.68828 \times 10^{-25}$ \\
\hline
\end{tabular}

In the table, $g_{\mu \nu}$ is the radius of the scattered particle, $M_{\mu \nu}$ is equal to the energy $(p)$ of the scattered particle, and $T_{\mu v}$ is equal to the gravitational force $(f)$ of the scattered particle.

The scattered particle of $7 \mathrm{TeV}$ proton-antiproton collision are incident in the corresponding detector, elastic collide or inelastic collide with the corresponding nucleus or outer electrons and transfer energy to the corresponding final state (Table.7, 8). 
Table 7.1 | Strong interaction data of the collision of the scattered particle in the detector

\begin{tabular}{l|c|c|l|c|c|c}
\hline \multirow{2}{*}{ particles } & $\lambda_{m}$ & $m$ & $v$ & $E, E_{c}$ & $F$ & $\phi_{\mu \nu}$ \\
\cline { 2 - 7 } & $10^{-12} m$ & $M e V$ & $m / s$ & $M e V$ & $N$ & $\mathrm{~m}^{-1}$ \\
\hline$e^{+}, e^{-}$ & 4.8526210703 & 0.510998928 & 299792457.921986 & 22398.512538 & $6.2736344 \times 10^{-38}$ & $6.455059 \times 10^{6}$ \\
$\mu_{\mu \mu}^{+}, \mu_{\mu \mu}^{-}$ & 4.8526210703 & 1.02199804 & 299792457.921325 & 44608.457752 & $1.2494452 \times 10^{-37}$ & $5.130592 \times 10^{6}$ \\
$p^{+}, p^{-}$ & 4.8527596967 & 938.272046 & 299788175.88 & 174609.88126 & $4.8906754 \times 10^{-37}$ & $1.053971 \times 10^{7}$ \\
$\tau_{\tau \tau}^{+}, \tau_{\tau \tau}^{-}$ & 4.8526235538 & 211.316581 & 299792381.2095 & 295028.69198 & $8.2635133 \times 10^{-37}$ & $8.904007 \times 10^{6}$ \\
$\mu^{+}, \mu^{-}$ & 4.8526238075 & 105.6583715 & 299792373.3725 & 140513.19142 & $3.9356559 \times 10^{-37}$ & $1.813595 \times 10^{7}$ \\
\hline
\end{tabular}

The scattered particles interact with the corresponding nucleus in the detector. The mass of the lead nucleus is approximately $3.441592 \times 10^{-24} \mathrm{~kg}$, the mass of the brass nucleus is approximately $1.05549906 \times 10^{-24} \mathrm{~kg}$, and the mass of argon is approximately $6.6353628 \times 10^{-25} \mathrm{~kg}$.

Table 7.2 Strong interaction data of the collision of the scattered particle in the detector

\begin{tabular}{c|c|c|c|c|c|c}
\hline$\varphi_{\mu \nu}$ & $R_{\mu \nu}$ & $r$ & $R$ & $r_{F}$ & $M_{\mu \nu}$ & $T_{\mu v}$ \\
\hline $\mathrm{m}$ & $\mathrm{m}^{-1}$ & $\mathrm{~m}$ & $\mathrm{~m}^{-2}$ & $\mathrm{~m}$ & $M e V, \rho_{p} / \mathrm{m}^{3}$ & $M e V, \rho_{p} / \mathrm{m}^{3}$ \\
\hline $9.25747 \times 10^{-15}$ & $1.291019 \times 10^{7}$ & $1.095208 \times 10^{-14}$ & $1.39456 \times 10^{21}$ & $3.78701 \times 10^{-11}$ & 18932.7938 & $3.37508 \times 10^{-26}$ \\
$7.358 \times 10^{-15}$ & $1.026118 \times 10^{7}$ & $8.704906 \times 10^{-15}$ & $1.39456 \times 10^{21}$ & $3.78701 \times 10^{-11}$ & 37706.19387 & $6.72174 \times 10^{-26}$ \\
$4.66054 \times 10^{-15}$ & $2.107941 \times 10^{7}$ & $5.513673 \times 10^{-15}$ & $4.52295 \times 10^{21}$ & $2.10283 \times 10^{-11}$ & 147592.5203 & $2.63108 \times 10^{-25}$ \\
$3.91901 \times 10^{-15}$ & $1.780801 \times 10^{7}$ & $4.636405 \times 10^{-15}$ & $4.5440 \times 10^{21}$ & $2.09795 \times 10^{-11}$ & 249378.9463 & $4.44559 \times 10^{-25}$ \\
$5.01827 \times 10^{-15}$ & $3.627189 \times 10^{7}$ & $5.936885 \times 10^{-15}$ & $7.22797 \times 10^{21}$ & $1.66344 \times 10^{-11}$ & 118771.6062 & $2.1173 \times 10^{-25}$ \\
\hline
\end{tabular}

In the table, $\varphi_{\mu \nu}$ is the phase difference of scattering particles from collision to decay. $M_{\mu \nu}$ is the energy transfer by the scattered particle to final state, which is very consistent with the data of ATLAS and CMS experiment, such as the energy range of final state in the table $(1,2)$.

Table $8.1 \mid$ Weak interaction data of the decay of the scattered particle in the detector

\begin{tabular}{l|c|c|l|c|l|l}
\hline \multirow{2}{*}{ particles } & $\lambda_{m}$ & $m$ & $v$ & $E, E_{c}$ & $F$ & $\phi_{\mu \nu}$ \\
\cline { 2 - 7 } & $10^{-12} m$ & $M e V$ & $m / s$ & $M e V$ & $N$ & $\mathrm{~m}^{-1}$ \\
\hline$e^{+}, e^{-}$ & 4.8526211675 & 0.510998928 & 299792454.920131 & 3564.40437 & $9.9835959 \times 10^{-38}$ & $1.409004 \times 10^{7}$ \\
$\mu_{\mu \mu}^{+}, \mu_{\mu \mu}^{-}$ & 4.8526211683 & 1.02199804 & 299792454.894038 & 7098.79821 & $1.9883135 \times 10^{-38}$ & $1.119901 \times 10^{7}$ \\
$p^{+}, p^{-}$ & 4.8580999405 & 938.272046 & 299623360.196 & 27001.0843 & $7.5627759 \times 10^{-38}$ & $2.235554 \times 10^{7}$ \\
$\tau_{\tau \tau}^{+}, \tau_{\tau \tau}^{-}$ & 4.8527192113 & 211.316581 & 299789426.417636 & 46777.5902 & $1.3102022 \times 10^{-37}$ & $1.936437 \times 10^{7}$ \\
$\mu^{+}, \mu^{-}$ & 4.8527292278 & 105.6583715 & 299789117.02207 & 22274.5267 & $6.2389069 \times 10^{-38}$ & $3.943446 \times 10^{7}$ \\
\hline
\end{tabular}

Table 8.2 | Weak interaction data of the decay of the scattered particle in the detector

\begin{tabular}{c|c|c|c|c|c|c}
\hline$g_{\mu v}$ & $R_{\mu \nu}$ & $r$ & $R$ & $r_{F}$ & $M_{\mu v}$ & $T_{\mu v}$ \\
\hline $\mathrm{m}$ & $\mathrm{m}^{-1}$ & $\mathrm{~m}$ & $\mathrm{~m}^{-2}$ & $\mathrm{~m}$ & $M e V, \rho_{p} / \mathrm{m}^{3}$ & $\mathrm{MeV}, \rho_{p} / \mathrm{m}^{3}$ \\
\hline $2.02096 \times 10^{-14}$ & $2.818008 \times 10^{7}$ & $2.020955 \times 10^{-14}$ & $1.39439 \times 10^{21}$ & $3.78723 \times 10^{-11}$ & 3564.40437 & $6.35413 \times 10^{-27}$ \\
$1.60629 \times 10^{-14}$ & $2.2398 \times 10^{7}$ & $1.60629 \times 10^{-14}$ & $1.39439 \times 10^{21}$ & $3.78724 \times 10^{-11}$ & 7098.79821 & $1.26548 \times 10^{-26}$ \\
$1.01742 \times 10^{-14}$ & $4.471108 \times 10^{7}$ & $1.017422 \times 10^{-14}$ & $4.39455 \times 10^{21}$ & $2.13333 \times 10^{-11}$ & 27001.0843 & $4.81338 \times 10^{-26}$ \\
$8.55542 \times 10^{-15}$ & $3.872874 \times 10^{7}$ & $8.555419 \times 10^{-15}$ & $4.52681 \times 10^{21}$ & $2.10193 \times 10^{-11}$ & 46777.5902 & $8.33887 \times 10^{-26}$ \\
$1.09552 \times 10^{-14}$ & $7.886893 \times 10^{7}$ & $1.095516 \times 10^{-14}$ & $7.19925 \times 10^{21}$ & $1.66675 \times 10^{-11}$ & 22274.5267 & $3.9708 \times 10^{-26}$ \\
\hline
\end{tabular}

In the table $g_{\mu \nu}$ is the radius of the scattering particles.

The unified phase field theory and curvature tensor equation based on the interaction of positive and negative magnetic poles naturally and perfectly describe the unified of the strong interaction, weak 
interaction, electromagnetic interaction and gravitational interaction during the collision and decay of high-energy particle. When the scattering particles collide with the detector nucleus or outer layer electrons, $\phi_{\mu v}$ represents the phase field curvature tensor of the scattered particle, $R_{\mu \nu}-\frac{1}{2} \varphi_{\mu \nu} R$ represents the differential geometric curvature tensor of the scattered particle, $\frac{r}{f_{\mu \nu} r_{F}^{2}} T_{\mu \nu}$ represents the curvature tensor of the gravitational field of scattered particle, and $\frac{r}{p_{\mu \nu} r_{F}^{2}} M_{\mu \nu}$ represents the curvature tensor of the electromagnetic (energy) field of scattered particle.

\section{Discussion}

It is assumed that the elementary particle is a magnetic poles field formed by the interaction of positive and negative magnetic pole (Fig.a). The positive magnetic pole ring at the core of the elementary particle condenses the negative magnetic pole into a magnetic poles field, and the interaction of the positive and negative magnetic pole drives the rotation of the elementary particle. In other words, the interaction between the positive magnetic pole ring and the negative magnetic pole field forms elementary particles that rotate continuously. The so-called elementary particle spin refers to the interaction motion of the continuously rotates elementary particle and the external energy field.

Free quark, gluon, and $\mathrm{W}$ and $\mathrm{Z}$ boson were not found in the universe and physics experiments. The high-energy particle based on the interaction of positive and negative magnetic pole do not need to be constructed with third-generation quark and gluon, and their decay does not require the mediation of $\mathrm{W}$ and $\mathrm{Z}$ intermediate boson. The interaction between elementary particles and external fields (elementary particles are each other's external fields) based on the interaction of positive and negative magnetic pole can explain black-body radiation experiment, photoelectric effect experiment, double-slit

experiment, $\beta$ decay experiment, various high-energy particle collision experiments, etc.

The interaction between elementary particles and the external field strictly follows the principle of conservation of energy, accelerating condenses energy, and decelerating releases energy. The inelastic collision of the elementary particles can cause two positive magnetic pole rings to merge, or can occur two positive magnetic pole rings fission. Using this interaction principle to explain the collision and decay of high-energy particle is more in line with physical reality. This article takes the European Large Hadrons Collider as an example to discuss related issues.

\section{Accelerating proton to condense more magnetic poles}

The composite particles cannot approach the speed $(c)$ of light in any gravitational field in the universe or on any particle accelerator that people make (will disintegrate when approaching the speed of light). Only elementary particles (the particles of one positive magnetic pole ring) can approach the speed of light. When the elementary particles accelerate, more negative magnetic pole (negative magnetic pole absorbed from the external field) will be condensed, and the number of magnetic pole in the positive magnetic pole ring will not increase, but the two positive magnetic poles will be closer 
together. Therefore, the interaction between the positive magnetic pole ring and the negative magnetic pole field is stronger. If the protons accelerate, its relativistic mass or energy keep increasing, the motion wavelength and radius keep decreasing, and related variables covariant. The motion mass of the elementary particle according to Einstein's mass-energy equation

$$
m_{m}=m / \sqrt{1-\left(v^{2} / c^{2}\right)}-m,
$$

Where $v$ is the equivalent velocity of the elementary particles, $c$ is the speed of light. The equivalent velocity $(v)$ of the elementary particle is equal to that the actual displacement velocity of the particle in the external field plus the equivalent velocity of the energy density of the external field. An external field with a certain energy density has a certain resistance to the movement of the particle, $c$ is the velocity $(v)$ of photons in absolute zero vacuum. The equivalent velocity of the elementary particle

$$
v=k / \sqrt{\lambda_{m}}, \quad \lambda_{m}=(k / v)^{2}
$$

Where $\lambda_{m}$ is the particle motion wavelength, $k=6.60402779758742 \times 10^{2}$ is the conversion constant between the particle motion wavelength and the particle equivalent velocity. When the actual displacement velocity of the particle in the external field is equal to zero, $v$ is the equivalent velocity of the energy density of the external field, $\lambda_{m}$ is the wavelength of the energy density of the external field or the motion wavelength of the particle. Newtonian kinetic energy equation based on the theory of relativity

$$
E=r \frac{1}{2}\left(m+m_{m}\right) v=m_{m} c^{2},
$$

Where $r$ is the correction value from 1 to 2 . Newtonian gravitational equation based on the theory of relativity

$$
F=\sqrt{G\left(m_{1}+m_{1 m}\right)\left(m_{2}+m_{2 m}\right) / r^{2}},
$$

There is a ratio between the gravitational force $(F)$ and the energy $(E)$ of the particle in the interaction between particles and external fields, for example, the ratio of the gravitational force $(F)$ to the energy $(E)$ of an electron is $1.78266326294376 \times 10^{-30}$. The free falling speeds of simultaneous particles of different masses are the same in the gravitational field. Therefore, the equivalent speeds of particles of different masses in the external field of same energy densities are also the same.

The relativistic mass $(M)$ of the elementary particle moving in an external field with a certain energy density can be divided into the core field mass in the positive magnetic pole ring and the gradient field mass outside the ring. The relativistic mass is not the rest mass. The rest mass is the mass when the particle is in an external field of absolute zero and there is no displacement. Although the rest mass of a proton is determined based on the relativistic mass, it is almost equal to its rest mass in an external field of absolute zero. The elementary particle spin magnetic poles field (or energy field) equation based on relativity 


$$
\begin{aligned}
& M=e \int_{i=1}^{i=\alpha}\left(\left(m+m_{m}\right) / e\right)\left((i+\delta)^{3}-i^{3}\right) d V \\
& =\left(m+m_{m}\right) \int_{i=1}^{i=\alpha}\left((i+\delta)^{3}-i^{3}\right) d V
\end{aligned}
$$

Where $e$ is the natural constant, which is the convergence limit constant of the elementary particle's positive magnetic pole ring; $i=1$ is the particle's positive magnetic pole ring wavelength, which is the initial value of the elementary particle gradient field; $i=\alpha$ is the elementary particle motion wavelength, which is the boundary value of the elementary particle gradient field. $\delta$ is the wavelength

difference of the elementary particle gradient field, $\int_{i=1}^{i=\alpha}\left((i+\delta)^{3}-i^{3}\right) d V=1 . d V$ is the gradient volume of the particle gradient energy field corresponding to the gradient wavelength difference. The equation (8) reflects the physical reality of the movement of the elementary particle, there is no ultraviolet divergence and infrared divergence, and no renormalization is required.

\section{The unified phase field theory and the curvature tensor equation of collision and decay of} high-energy particle

The so-called unified phase field theory is the theory that the differential geometric ${ }^{30,31,32,33}$ variables and physical variables are covariant, and to realize the unified of the gravity, the electromagnetic force, the strong force, and the weak force in the interaction of the magnetic poles field of particles based on the theory of relativity. There four forces are all produced by the interaction of positive and negative magnetic poles, which are four manifestations of the interaction of positive and negative magnetic poles. The negative magnetic pole acts as a mediator in these four interactions, and no other mediator is needed. This is more in line with physical reality.

The gravity refers to the cohesive force of the particle magnetic poles field (the gravitational field) to its magnetic poles is gravitational force. The high-energy proton and antiproton act as gravitational fields for each other, are in gradient magnetic poles field of each other, have a strong attractive force, and the gravitational force increases as the distance between them decreases, when its collide. Scattering particles are incident in the gradient magnetic poles field of the crystal lattice of the relevant element, and are attracted by the nucleus gravity and enter the corresponding gradient energy field of the nucleus. The gravitational force of the scattering particles increases as the distance from the nucleus decreases.

The electromagnetic force refers to the spin force of the particle gradient magnetic pole field in the external field (the gravitational field). The high-energy proton and antiproton act as gravitational fields for each other and spin in each other's gradient magnetic pole field to generate spin electromagnetic force (or energy) when its collide. The gradient magnetic pole field of the scattering particles spins in the atomic (or outer electron) gradient magnetic pole field to generate spin electromagnetic force (or energy).

The strong force refers to the maximum interaction force when high-energy particles collide. The strong force is the maximum interaction force when that scattering particles collide with atomic gradient magnetic pole field or the outer electron.

The weak force refers to the interaction force when the positive magnetic pole ring of the high-energy particles decays in the process of the interaction between the high-energy particle and the external field. For example, a positive pole ring of high-energy particle splits into a positive pole ring of leptons and a 
positive pole ring of neutrinos when its decays, that is, high-energy particles decay into leptons and neutrinos.

The phase of the elementary particle can be obtained according to the motion wavelength of its

$$
r_{\varphi}=\alpha \lambda_{m} / 2 \pi
$$

where $\lambda_{m}$ is the motion wavelength of the elementary particle, $r_{\varphi}$ is the phase of the elementary particle, or the radius $r$ of spin phase field of the elementary particle. The difference of the phase before and after the particle moves along the space-time curve

$$
\varphi_{\mu v}=g^{\mu v}\left(\partial q_{\mu v} / q\right)
$$

where $q$ is the velocity of particle, and the metric $g_{\mu v}=e_{\mu} \cdot e_{v}$.

The interactions of particle and external field can be described with tensor. The so-called tensor refers to a multi-stress vector function or a multi-linear function of point, line, area, and volume with a certain energy density. Particle's spin phase field is a gradient energy field, and can be described with tensor of point, vector, area and volume, which are represented as scalar, first-order tensor, second-order tensor, third-order tensor, etc. In addition to scalar, other tensors belong to gradient tensor, and the average is taken as its unit tensor.

The interaction between particles and the external field can be represented by a tensor and each tensor is covariant with the space-time curvature of the particle motion. The particle with a certain radius $(r)$ represent as the momentum-energy tensor $\left(p_{\mu v}\right)$, the gravitational momentum-energy tensor $\left(f_{\mu v}\right)$, the phase difference momentum-energy tensor $\left(M_{\mu \nu}\right)$; The external field with a certain radius $\left(r_{F}\right)$ represent as the gravitational field phase difference momentum-energy tensor $\left(T_{\mu \nu}\right)^{34,35}$, Ricci curvature tensor $\left(R_{\mu v}\right)$, the phase field curvature tensor $\left(\phi_{\mu v}\right)$, the gravitational field curvature tensor $\left(\frac{r}{f_{\mu \nu} r_{F}^{2}} T_{\mu \nu}\right)$, the electromagnetic field curvature tensor $\left(\frac{r}{p_{\mu \nu} r_{F}^{2}} M_{\mu \nu}\right)$. The curvature tensor equation of the interactions between spin phase field of particles and spin phase field of external field, which is the curvature tensor equation of unified phase field

$$
\phi_{\mu \nu}=R_{\mu \nu}-\frac{1}{2} \varphi_{\mu \nu} R=\frac{r}{f_{\mu \nu} r_{F}^{2}} T_{\mu \nu}=\frac{r}{p_{\mu \nu} r_{F}^{2}} M_{\mu v},
$$

Where $\varphi_{\mu \nu}$ is the phase difference metric and is used to calculates the curvature tensor of the particle's phase difference. Replace $\varphi_{\mu \nu}$ with the phase metric or Riemann metric $\left(g_{\mu \nu}\right)$ to calculate the curvature tensor of the particle. $r$ is the radius of particle's spin phase field, $1 / r_{F}{ }^{2}$ is Gauss curvature $(K)^{36}, r_{F}$ is the radius of spin phase field of external field (gravitational field), 
$R=2 K=2 / r_{F}^{2}$ is the scalar of Ricci curvature ${ }^{37,38}, R_{\mu \nu}=R \varphi_{\mu \nu}$ is Ricci curvature tensor of the particle's phase difference, $\mu$ and $v$ is the tensor subscript (the basis vector of local coordinate system, which represents the radius of which before and after particles is translated along space-time curve). The particle's momentum-energy tensor $p_{\mu \nu}=\rho_{p} V$, namely the momentum-energy density $\left(\rho_{p}\right)$ of particle multiply by the particle volume $(V)$. The particle's gravitational momentum-energy tensor $f_{\mu v}=\rho_{f} V$, namely the gravitational momentum-energy density $\left(\rho_{f}\right)$ of particle multiply by the particle volume $(V)$.

The curvature tensor equation of unified phase field describes the space-time curve or Ricci curvature flow of particle motion. The phase difference momentum-energy tensor $\left(M_{\mu \nu}\right)$ of the particle is relativistic Lagrangian density of the particle, and equivalent to the Lagrangian density $\left(L_{F}\right)$ described by the Yang-Mills gauge field equation ${ }^{39} \cdot M_{\mu \nu}$ of the curvature tensor equation of unified phase field can be expressed in the Lagrangian function of energy, wave function and gauge field of particles motion. The Lagrangian function of Einstein's mass-energy equation

$$
L_{E}=E \frac{i \partial}{\partial r}=\left(m / \sqrt{1-\left(v_{t 1} / c\right)^{2}}-m\right)^{2}-\left(m / \sqrt{1-\left(v_{t 2} / c\right)^{2}}-m\right)^{2}
$$

Where $E$ is the energy of the colliding particle. $\frac{i \partial}{\partial r}$ is the particle phase vector between the colliding particle and the scattering particle, is the ratio of the phase difference between the colliding particle and the scattering particle to the phase of the colliding particle, and which $i$ is a complex number. $v_{t 1}, v_{t 2}$ are respectively the velocity of the colliding particle and the scattering particle. The energy of the particle is covariant with the phase difference of the particle phase field. Lagrangian function of Schrodinger particle differential motion wave function based on the theory of relativity

$$
L_{\psi}=E \psi \frac{i \partial}{\partial r}=i \beta h_{m} \frac{\partial}{\partial t} \psi(\vec{r}, t)=\left[-\frac{\beta^{2} h_{m}^{2}}{2 m+m_{m}} \nabla^{2}+V(\vec{r}, t)\right] \psi(\vec{r}, t)
$$

Where E$\psi$ is the energy wave function of the colliding particle. $\beta$ is the factor of relativistic kinetic energy operator, $\beta=\frac{p}{k h_{m}} \cdot p$ is the kinetic energy of the particle, $k=\frac{4 \pi}{\alpha \lambda}, \lambda$ is the wavelength of the particle motion, and $\alpha$ is the fine structure constant. $h_{m}$ is the rest mass constant based on the wavelength of the particle motion, $h_{m}=5.45025577174353 \times 10^{-65} \mathrm{~kg} \cdot \mathrm{m}^{3}$. 
$-\frac{\beta^{2} h_{m}^{2}}{2 m+m_{m}} \nabla^{2}$ is the particle kinetic energy operator $(\hat{T}), \nabla^{2}$ is the Laplacian operator. $V(\vec{r}, t)$

is the particle potential energy, $\vec{r}$ represents the potential energy vector. The wave function of the particle is covariant with the phase difference of the particle phase field. Lagrangian Density of Yang-Mills gauge field equation ${ }^{39}$ in the First Order Form

$$
\begin{aligned}
& L_{F}=F_{E} \frac{i \partial}{\partial r}=\frac{1}{4} F_{\mu v} \cdot F^{\mu v}-\frac{1}{4} F_{v \mu} \cdot F^{v \mu} \\
& =\frac{1}{4} F_{\mu v} \cdot F^{\mu v}-\frac{1}{2} F_{v \mu} \cdot\left(\partial^{\mu} A^{v}-\partial^{v} A^{\mu}+g A^{\mu} \times A^{v}\right)
\end{aligned}
$$

Where $F_{E}$ is the energy field of the colliding particle. $F_{\mu \nu}, F_{v \mu}, A_{\mu}$ and $A_{v}$ are respectively independent coordinates, which represents the spin vector, and the dot product and cross product represent the operation between them. The gauge field is the spin gradient phase field in which the particle interacts with the external field. The energy of the gauge field is covariant with the phase difference of the particle phase field. Yang-Mills Lagrangian Density $\left(L_{F}\right)$ is the energy $\left(\frac{i \partial}{\partial r} m_{m} c^{2}\right)$ field of particle motion mass covariant with the particle phase difference.

The Lagrangian function of gravitational field phase difference momentum-energy tensor $\left(T_{\mu \nu}\right)$ of Newtonian gravitational equation based on the theory of relativity (see equation (7))

$$
L_{T}=F \frac{i \partial}{\partial r}=\sqrt{G\left(m_{1}+m_{1 m}\right)\left(m_{2}+m_{2 m}\right) / r_{F 1}^{2}}-\sqrt{G\left(m_{1}+m_{1 m}\right)\left(m_{2}+m_{2 m}\right) / r_{F 2}^{2}}
$$

Where $F$ is the gravitational force of the colliding particle. $r_{F 1}, r_{F 2}$ are respectively the gravitational field radius of the colliding particle and the scattering particle. The gravity of a particle is covariant with the phase difference of the particle phase field.

The curvature tensor equation of unified phase field obeys the principle of relativity, realizes the unity of Einstein's special theory of relativity and general relativity, and profoundly reveals the physical reality of the interaction between particles and external fields. The Lagrangian function of Einstein's mass-energy equation, the Lagrangian function of Schrodinger particle differential motion wave function based on the theory of relativity, the Lagrangian density of Young-Mills gauge field equation, and the particles phase difference momentum-energy tensor of the curvature tensor equation of unified phase field is completely consistent, namely $L_{E}=L_{\psi}=L_{F}=M_{\mu v}$, realizes the unity of relativity and quantum mechanics.

\section{Methods}

\section{The high-energy field produced by the collision of high-energy proton and antiproton}

The high-energy proton and antiproton condenses a large number of magnetic poles (or energy) in the process of acceleration, and release the corresponding magnetic poles when collision. High-energy 
proton-antiproton collides, and the released magnetic pole is superimposed with a certain external field magnetic poles to form the energy field of high-energy particle collision.

High-energy proton is a gradient magnetic poles field or gradient energy field. Most of proton and antiproton elastic collide and a few proton and antiproton inelastic collide in the proton and antiproton collides. The instantaneously released magnetic poles or energy $\left(E_{f}\right)$ in the high-energy proton (or elementary particles ) inelastic collide is equal to the energy $\left(E_{c}\right)$ when its collision subtracts the energy $\left(E_{d}\right)$ when its decay, $E_{d}=E_{c} / 2 \pi, E_{f}=E_{c}-E_{d}$.

The European Large Hadrons Collider (LHC) uses high-energy proton beams to collide with high-energy antiproton beam to increase the probability of high-energy proton and antiproton collisions. When the high-energy proton beams collides with the anti-proton beam, the leading proton and anti-proton inelastic collide, which will produce new particles, release a large amount of energy, and form an energy field. If the subsequent proton and antiproton inelastic collisions, the released energy will be superimposed with the external energy field to form a high-energy field of collision. The proton and antiproton elastic collide, release a certain amount of energy, and run away along the beam tube.

When protons and antiproton beams collide, the high-energy field of the collision is a gradient energy field whose energy density decreases from the inside to the outside, or an energy field whose energy density wavelength increases from the inside to the outside. The energy of the energy field is a spherical wave radiating outward. This is the magnetic pole wave.

High-energy proton and antiproton beams collide once, which is a collision event. There is about $10^{11}$ proton in each beam, which is compressed into a filament with a diameter of $16 \mu \mathrm{m}$ and a length of 7 $\mathrm{cm}$. About 23 pairs of proton inelastic collide in a collision event. The energy of the collision energy field is less than the superposition state of the energy released by the collision of 23 pairs of proton, because the magnetic poles wave diffusion speed of collision energy is greater than the motion speed of high-energy proton.

\section{Collision and decay of high-energy proton and antiproton}

The so-called elastic collision refers to the collision between the gradient magnetic poles field of two protons (or elementary particles) with opposite spin directions, not necessarily equal energy and deviate the collision line. The so-called inelastic collision, one refers to the merging or fission of the positive magnetic pole ring that the collision between two protons (or elementary particles) with opposite spin directions and equal energy (Fig.b,c,d); the second refers to positive magnetic pole ring fission with small energy that the collision between two high-energy elementary particles with opposite spin directions and unequal energy; the third refers to the deep collision between the high-energy elementary particles and the atomic nucleus or outer electrons. The so-called merging of positive magnetic pole rings refers to that the positive magnetic pole rings of two elementary particles merged into positive magnetic pole ring of a particle. For example, the positive magnetic pole rings of protonium is composed of the positive magnetic pole rings of proton and antiproton colliding and merging (Fig.b,c). The so-called positive magnetic pole ring fission refers to that a positive magnetic pole ring of the elementary particle splits into positive magnetic pole ring of a lepton and positive magnetic pole ring of a lepton neutrino (Fig.b,d), or a positive magnetic pole ring of 
particle-pair splits into two positive magnetic pole rings of the elementary particles with equal energy and opposite spins(Fig.b,c).
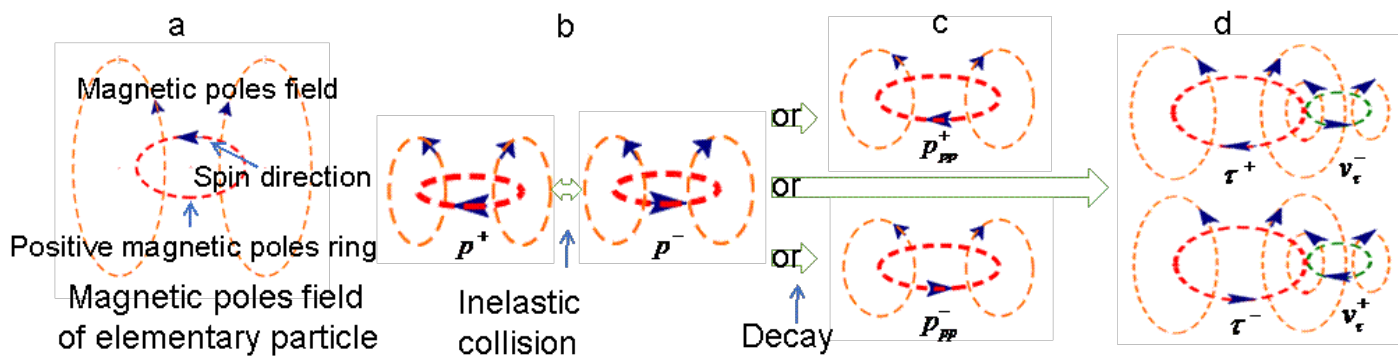

Figure | The magnetic poles field of the elementary particle and the collision and decay of high-energy proton and antiproton. (a) The positive magnetic pole ring of the elementary particle drives the negative magnetic pole field to spin in the external field, forming a magnetic poles field that conforms to the right-hand or left-hand rule. (b) Inelastic collision between proton and antiproton. (c) Inelastic collisions of proton and antiproton may produce protonium or anti-protonium. (d) Inelastic collisions between proton and antiproton may produce tau, anti-tau and tau-neutrinos, anti-tau-neutrinos.

Inelastic collisions of three levels of high-energy particle occur successively in the high-energy proton collision energy field.

The inelastic collision of first-level is inelastic collision of the high-energy proton and antiproton. The collisions of high-energy proton and antiproton inelastic, one is that protonium may be produced, which energy is the sum of the energy of two high-energy protons when its decays (Fig.b,c), and other is that its may produce tau, anti tau and tau-neutrinos, anti tau-neutrinos (Fig.b,d). The spin direction of Tau is the same as that of proton, and the spin direction of tau-neutrino is opposite to that of proton. The sum of the energy of tau and tau-neutrinos is equal to the energy of proton when it decays. Tau neutrinos scatter as the collision energy field expands.

The inelastic collisions of second-level are the inelastic collisions of high-energy protonium and the inelastic collisions of high-energy tau and anti-tau. The inelastic collisions of high-energy protonium (or the collision between protonium and incident proton), produce proton and antiproton with relativistic energy, which are scattered as the collision energy field expands. The inelastic collision of high-energy tau and anti-tau may produce tauium, whose energy is the sum of the energy of two taus when they decay. The tauium has a relativistic mass and scatters as the collision energy field expands. The inelastic collision of high-energy tau and anti-tau (or the collision between tau, anti-tau and incident proton) may produce muon, anti-muon and muon neutrino, anti-muon neutrino, which will be scattered as the collision energy field expands. The direction of muon spin is the same as tau, and the direction of muon neutrino spin is opposite to that of tau. The sum of the energy of muon and muon neutrino is equal to the energy of tau when it decays. If in a high-energy proton and antiproton collision event, there are two the inelastic collision of high-energy tau and anti-tau (or the collision between tau, anti-tau and incident proton), it may produce the so-called "golden decay channel".

The inelastic collision of third-level is the inelastic collision of high-energy muon and anti muon. The inelastic collision of high-energy muon and anti muon may produce muonium, whose energy is the sum of the energy of the two muons when they decay. The muonium has a relativistic mass, and scatters as the collision energy field expands. The inelastic collisions of high-energy muon and anti-muon (or collision between muon and proton or muon and tau) may produce electron, positron and electron-neutrino, positron-neutrino, which will be scattered as the collision energy field expands. The spin direction of electron is the same as that of muon, and the spin direction of electron-neutrino is 
opposite to that of muon. The sum of the energy of electron and electron-neutrino is equal to the energy of the muon when it decays.

When the high-energy particle beam collide, if the scattered particles have "simultaneity", injection phenomenon produced. The ratio of energy to volume of tauium Incident to the hadron calorimeter is relatively large, and tracks curvature is small, therefore, collinear scattering occurs.

The inelastic collision of the three levels of high-energy particle reveals the production mechanism of the protonium, tauium, muonium, positronium, the third-generation lepton and neutrino, and jets. The three generations of neutrinos all have the speed of light after they are produced. This is because when a neutrino has produced, energy density and the positive magnetic pole density of the neutrino are the same as the leptons, but mass and radius of the neutrino are smaller than that of the lepton. Relatively speaking, the interaction force of between the positive magnetic poles ring of the neutrino with its negative magnetic pole field is greater than that of the lepton. The rest mass of the electron-neutrino is about $1.319849 \mathrm{eV}$, the rest mass of the muon-neutrino is about $0.272903167 \mathrm{keV}$, the rest mass of the tau-neutrino is about $4.58942073879939 \mathrm{keV}$. The speed of neutrinos is equal to the speed of photons, is about $299792457.9999999335677253410648 \mathrm{~m} / \mathrm{s}$. Troitzk ${ }^{40,41}$ experiments obtained the upper limit of mass less than $2.05 \mathrm{eV}$. In 2017, the KATRIN experiment ${ }^{42}$ in Germany limited the neutrino mass from the end of the decay electron spectroscopy, obtained neutrino mass upper limit is $1 \mathrm{eV}$.

\section{Collision and decay of scattered particle in the detector}

The scattered particle produced by the collision of high-energy particle are scattered with the expansion of the collision energy field. The scattered particles are detected by the detectors. Tack the CMS detector as example ${ }^{43}$. The central feature of the CMS apparatus is a superconducting solenoid of $6 \mathrm{~m}$ internal diameter, which provides a magnetic field of $3.8 \mathrm{~T}$. Within the field volume are a silicon pixel and strip tracker, a lead tungstate crystal electromagnetic calorimeter (ECAL), and a brass/scintillator hadron calorimeter (HCAL). Muon is measured in gas-ionization detectors embedded in the steel flux-return yoke. Extensive forward calorimeters complement the coverage provided by the barrel and endcap detectors.

The scattered particle have relativistic energy and motion wavelength when they leave the collision energy field and enter the detector energy field, which is a spin magnetic poles field with a certain radius. The scattering particles take the shortest path in the element lattice magnetic field and the detection element "lattice" magnetic field. Different particles interact with different strengths in different detectors. The larger the energy of the scattered particle, the smaller it's volume, and vice versa. Scattering particle can be divided into fermion and boson. Both fermion and boson have a ratio of energy to volume. A particle with a large ratio of energy to volume has a small Gaussian curvature in the same magnetic field, and vice versa. The energy density wavelength of a uniform magnetic field of $3.8 \mathrm{~T}$ is equivalent to the infrared photon wavelength, which is about $7.5 \times 10^{-6} \mathrm{~m}$.

Various scattering particles interact with the $3.8 \mathrm{~T}$ uniform magnetic field, or interact with the element gradient energy field. Particles with a large ratio of energy to volume will be incident in the element deep gradient energy field and elastic collide or inelastic collide with the outer electrons or the nucleus in the element. The kinetic energy of the scattering particle is larger, and the kinetic energy of the nucleus or outer electrons is smaller. The scattering particle will transfer the energy () to the nucleus or outer electrons, or transferred to hadrons shower or electromagnetic shower, when inelastic collide.

Various scattered particle are incident in the multi-layer silicon microchip lattice of the inner detectors 
and elastic collide with the semiconductor electrons, thereby generating signals and showing tracks. The three types of neutrinos have a large ratio of energy to volume and no tracks in various detectors; the ratio of energy to volume of muonium is large, and the tracks curvature is small, similar to photon; the ratio of energy to volume of proton and tauium is large, and the tracks curvature is relatively small, tauium similar to photon; the ratio of energy to volume of muon is small, and the tracks curvature is slightly larger (or show a weak neutral current); the ratio of energy to volume of electron is small, and the tracks curvature is large.

The electrons are incident in the lead tungstate crystal, and elastic collide or inelastic collide with the outer layer electrons in the element to produce electromagnetic showers, which become free electrons. The muonium are incident in the lead tungstate crystal and inelastic collide with the outer electrons in the element to produce electromagnetic showers, and decays into muon and anti muon. Muons inelastic collide with outer electrons and decay into electrons and electron-neutrinos. The decay vertex of muon is the produces vertex of electron and electron-neutrino. Electron with a certain energy collide with the outer electrons, which may produce positronium (annihilated as gamma photons), and then collide with the outer electrons to become free electron. Proton, tauium and muon are incident in the lead tungstate crystal, and elastic collide with the outer electrons and the nucleus, releasing part of the magnetic poles, thereby generating signal, showing tracks, and passing through the electromagnetic calorimeter.

The proton and tauium are incident in the hadron calorimeter and elastic collide or inelastic collide with the nucleus of the brass/scintillator, thereby generating signals and showing tracks. The scattered proton elastic collide or inelastic collides with the nucleus of the brass/scintillator will produce hadrons showers. The scattered protons inelastic collide with nucleus, causing a certain angle of refraction, and finally decay into baryons and precipitate in the brass/scintillator crystal. Baryons are proton with a certain relativistic mass in a certain energy field. Tauium elastic collide or inelastic collide with the nucleus of the brass/scintillator, produce meson shower, and finally decays into the corresponding meson, which is precipitate in the brass/scintillator crystal. The muon are incident in the brass/scintillator crystal and elastic collides with the outer electrons and the nucleus, releasing part of the magnetic poles and passing through the hadron calorimeter.

The muon are incident in the gas chamber of the muon detector and elastic collides with the mixed gas of argon and carbon dioxide to ionize the gas, thereby generating signal and showing tracks. The kinetic energy of muon is determined according to the curvature of muon's tracks in the gas chamber. Finally, muon decays into electrons and electron-neutrinos, and the electrons are precipitate in the mixed gas of argon and carbon dioxide.

\section{References}

[1] M. Gell-Mann, Phys.Rev. 125, 1067-1084(1962).

[2] R.R Feynman, Murray Gell-Mann, G. Zweig, Phys.Rev.Lett 13, 678-680(1964).

[3] Gell-Mann M. Phys. Lett8,214(1964).

[4] Zweig G. CERN-TH-412,401 (1964).

[5] Schwinger, J. A theory of the fundamental interactions. Ann.Phys. 2(407), 34(1957).

[6] C. N. Yang and R. L. Mills. Isotopic spin conservation and generalized Gauge invariance. The Physical Review 95, $631(1954)$.

[7] P. W. Higgs, Phys. Lett. 12,132(1964); P.W Higgs, Phys. Rev. Lett. 13,508(1964).

[8] R. Brout, F Englert, Phys. Rev. Lett. 13,321(1964).

[9] G. S. Guralnik, C. R. Hagen, T.w.B. Kibble, Phys. Rev. Lett. 13,585(1964). 
[10] Weinberg, S. A model of leptons. Physical review letters. 19(21), 1264(1967).

[11] Gross, D. J., Wilczek, F. Ultraviolet behavior of non-abelian gauge theories. Physical Review Letters. 30(26), 1343-1346(1973).

[12] T. D. Lee and W. C. Wick, Phys. Rev. D 9,2291(1974).

[13] J. C. Collins and M. J. Perry,Phys. Rev. Lett 34,1353(1975).

[14] B. A. Freedman and L. D. McLerran, Phys. Rev. D 16,1161(1977).

[15] B. A. Freedman and L. D. McLerran, Phys. Rev. D 17,1109(1978).

[16] E. V. Shuryak, Phys. Rep 61,71(1980).

[17] F. J. Hasert, et al., Observation of neutruno-like interactions with muon, Phys. Lett. B46,138(1973).

[18] T. Eichten et al., Phys. Lett. B46,138(1973); F. J. Hasert et al., B73 (1974).

[19] Aubert J J. et al, Experimental Observation of a Heavy Particle-J[J]. Physical Review Letters, 33(23), 1404-1406(1974).

[20] Augustin J E. et al, Discovery of a Narrow Resonance in E+E-Annihilation[J]. Physical Review Letters, 33(23),1406-1408(1974).

[21] S. W. Herb, D. C. Horn, L. M. Ledennan, J. C. Sens,H. D. Snyder, J. K. Yoh, J. A. Appel, B. C. Brown, C, N.Brown, W. R. Innes, K. Ueno, T. Yamanouchi, A. S. Ito, H. Jdstlein, D. M. Kaplan, and R. D. Kephart, Phys.Rev. Lett. 39,252-255(1977).

[22] Barber, D. P., Becker, U., Benda, H. et al. Discovery of three-jet events and a test of quantum chromodynamics at PETRA. Physical Review Letters. 43(12), 830(1979).

[23] Clark, A. G. Latest results from the UA2 experiment at the CERN anti-p p collider: Hadron jets,W--> e nu,Z0--> e+ e,1st Asia-Pacific Physics Conference,vol.1,135-180(1983).

[24] F. Abe, et al (CDF Collaboration),Phys. Rev. Lett 74 ,626(1995).

[25] S. Abachi, et al, (DØ Collaboration), Phys. Rev. Lett. 74,2632(1995).

[26] DONUT Collaboration, Phys. Lett B 504,218-224(2001).

[27] ATLAS Collaboration. Observation of a new particle in the search for the Standard Model Higgs boson with the ATLAS detector at the LHC. Phys. Lett. B, 716:1,(2012).

[28] CMS Collaboration. Observation of a new boson at a mass of $125 \mathrm{GeV}$ with the CMS experi- ment at the LHC. Phys. Lett. $\mathrm{B}, 716: 30,(2012)$.

[29] Peter J. Mohr, Barry N. Taylor, and David B. Newell, Rev. Mod. Phys., Vol. 84, No. 4, October-December 2012.

[30] Riemann, B. Ueber die Hypothesen. welche der Geometrie zu Grundeliegen.Springer(1854).

[31] Riemann, B. On the Hypotheses which lie at the Bases of Geometry. Birkhäuser, (translated by William Kingdon Clifford. Nature, Vol. VIII. Nos. 183, 184, pp. 14-17, 36, 37) (2016).

[32] E. B. Christoffel. Ueber ein die Transformation homogener Differentialausdrücke zweiten Grades betreffendes Theorem. JOURNAL FUR DIE REINE UND ANGEWANDTE MATHEMATIK. No.70, 241-245(1869).

[33] Levi-Civita, M. T. Nozione di parallelismo in una varieta qualunque e conseguente specificazione geometrica della curvatura Riemanniana. Rendiconti del Circolo Matematico di Palermo (1884-1940), 42(1), 173-204(1916).

[34] Einstein, A. The foundation of the general theory of relativity. Annalen der Physik. 49(7), 769-822(1916).

[35] Einstein, A. Erklarung der Perihelionbewegung der Merkur aus der allgemeinen Relativitatstheorie. Sitzungsber. preuss. Akad. Wiss. vol. 47, No. 2, 831-839(1915).

[36] Gauss, C. F. Disquisitiones generales circa superficies curvas[M]. Vol.1.Typis Dieterichianis(1828).

[37] Ricci, M. M. G., Levi-Civita, T. Méthodes de calcul différentiel absolu et leurs applications. Mathematische Annalen. 54(1-2), 125-201(1900).

[38] M. M. G. Ricci. Sui parametri e gli invarianti delle forme quadratiche differenziali. Annali di Matematica Pura ed Applicata. No.1, 1-11(1886).

[39] C. N. Yang and R. L. Mills. 1954 Isotopic spin conservation and generalized Gauge invariance. The Physical Review 95,631 
[40] V. Lobashev. et al. Nucl. Phys. A719, 153c(2003).

[41] V. N. Aseev. et al. Phys. Rev. D84, 112003(2011).

[42] Lutz, Bornschein., Beate, Bornschein. et al. Status of the Karlsruhe Tritium Neutrino Mass Experiment KATRIN. Fusion Science and Technology. No.4, 485-490(2017).

[43] S. Chatrchyan, et al., The CMS experiment at the CERN LHC, JINST 3 (2008) S08004, http://dx.doi.org/10.1088/1748-0221/3/08/S08004.

\section{Acknowledgements}

The experimental data provided by ATLAS Collaboration and CMS Collaboration supports the research in this article.

\section{Author contributions}

This paper was independently researched and completed by the author.

\section{Original image file}

Figure | The magnetic poles field of the elementary particle and the collision and decay of high-energy proton and antiproton.

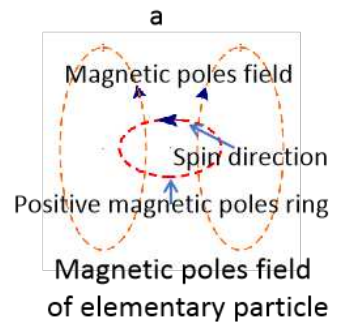

(a) The positive magnetic pole ring of the elementary particle drives the negative magnetic pole field to spin in the external field, forming a magnetic poles field that conforms to the right-hand or left-hand rule.

b

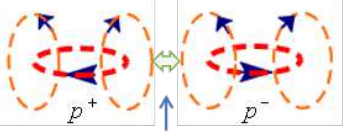

Inelastic collision

(b) Inelastic collision between proton and antiproton magnetic poles fields.

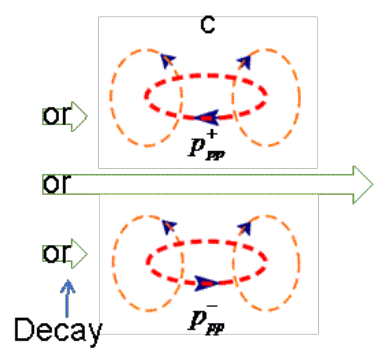

(c) Inelastic collisions of proton and antiproton may produce protonium or anti-protonium. 


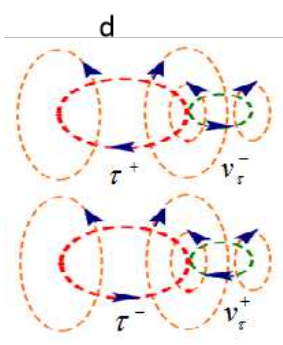

(d) Inelastic collisions between proton and antiproton may produce tau, anti-tau and tau-neutrinos, anti-tau-neutrinos. 